\title{
BOUNDEDNESS AND SURJECTIVITY IN NORMED SPACES
}

\section{OLAV NYGAARD}

Received 24 January 2001 and in revised form 6 June 2001

We define the $\left(w^{*}\right.$-) boundedness property and the $\left(w^{*}\right)$ surjectivity property for sets in normed spaces. We show that these properties are pairwise equivalent in complete normed spaces by characterizing them in terms of a category-like property called $\left(w^{*}\right.$-) thickness. We give examples of interesting sets having or not having these properties. In particular, we prove that the tensor product of two $w^{*}$-thick sets in $X^{* *}$ and $Y^{*}$ is a $w^{*}$-thick subset in $L(X, Y)^{*}$ and obtain as a consequence that the set $w^{*}$-exp $B_{K\left(l_{2}\right) *}$ is $w^{*}$-thick.

2000 Mathematics Subject Classification: 46B20, 46B25, 28A33, 30H05.

1. Introduction. The following question is fundamental in the theory of linear operators: given two topological vector spaces $U$ and $V$. Suppose we are given a linear continuous operator $T: U \rightarrow V$ and suppose we can show that the range of $T$ contains a certain set $A \subset V$. Are there properties $(S)$ such that the following is true: if $A$ has the property $(S)$, then $T$ must be onto?

In one dimension the following is of course true: suppose $A \subset V$ contains one point different from the origin. Then $T U \supset A$ implies $T U=V$. In arbitrary $n$-dimensional spaces $V$ the theorem goes like this: suppose $A \subset V$ contains $n$ independent vectors. Then $T U \supset$ A implies $T U=V$.

Thus, the question is easy in finite-dimensional spaces. When $V$ does not have finitely many dimensions, the question is not easy. The problem is, naively spoken, that operators may very well have dense range without being onto. But from classical theorems we know something, for example, when $V$ is a normed space and $U$ is a Banach space, it was shown already by Banach in the twenties that if $A \subset V$ is of second (Baire-) category in $V$, then $T U \supset A$ implies $T U=V$.

However, there are examples of "smaller" sets than second category sets which allow one to draw the conclusion that the operator is onto. An example of such a situation is provided by taking $V=\ell_{\infty}$ and $A$ as the set of $0-1$ sequences (this follows as a special case of Seever's theorem [3, page 17]).

Suppose that the set $A \subset V$ has the surjectivity property if "onto $A$ implies onto $V$." Since, by linearity, also the symmetric, convex hull of $A$ must be contained in the range of $A$, we may assume $A$ to be symmetric and convex. On the other hand, the theorems are most useful when $A$ is "small," as in the $\ell_{\infty}$ situation. Also, by passing to quotients, $A$ has the surjectivity property if "onto $A$ implies onto $V$ for every injection."

Again, speaking naively and intuitively, by continuity, $T$ maps every convergent net in $U$ to a convergent net in $V$. So if $T U$ is dense, but $T$ is not onto, that must be because there are limits in $V$ that cannot be reached by the convergent nets which $T$ produces in $V$, from nets in $U$. 
When $U$ and $V$ are Banach spaces, a necessary and sufficient condition was given for a bounded set $A$ to have the surjectivity property, by Kadets and Fonf [14, Proposition 1].

TheOrem 1.1 (Kadets-Fonf [14]). Let $V$ be a Banach space and $A \subset S_{V}$. Then the following statements are equivalent:

(a) for any Banach space $U$ and any bounded linear operator $T: U \rightarrow V$ such that $T U \supset A$, one has $T U=V$;

(b) for every representation of $A$ as the union of an increasing sequence of sets, $A=\cup_{i=1}^{\infty} A_{i},\left(A_{i} \uparrow\right)$, there is an index $j$ such that

$$
\inf _{f \in S_{V^{*}}} \sup _{v \in A_{j}}|f(v)|>0
$$

(i.e., $A_{j}$ is a norming set for $V^{*}$, see Definition 2.1).

We call the property in statement (b) in the Fonf-Kadets theorem "thickness." Here we show that boundedness is not needed in their theorem and we show that if (b) fails, then there is a Tauberian injection onto $A$, but not onto $Y$ (see Theorem 4.2). The latter fact is obtained by using the construction of Davis, Figiel, Johnson, and Pełczyński [1].

We also extend the main result in [18] to unbounded sets. More precisely, we have the following theorem.

Theorem 1.2. Let $U$ and $V$ be Banach spaces. Suppose $A \subset V$ has the surjectivity property and suppose a family of linear continuous operators from $V$ into $U$ which is pointwise bounded on A is given. Then this family is uniformly bounded (i.e., bounded in $\mathscr{L}(V, U))$. Moreover, if A does not have the surjectivity property, then there is a sequence of operators which is pointwise bounded on $A$, but not bounded as a subset of $\mathscr{L}(V, U)$.

Thus, boundedness conclusions and surjectivity conclusions can be drawn from the common property (thickness).

Next, we study the situation when $U$ and $V$ are duals of normed spaces, equipped with weak star topologies. Again surjectivity and boundedness are connected and this time to a weaker thickness property. Combined with a theorem of H. Shapiro, this will have, as an immediate corollary, the following new theorem in complex function theory: suppose $T$ is a weak-star continuous linear operator from a dual Banach space $U$ into $H^{\infty}(D)$ such that TU contains the Blaschke products. Then $T$ is onto $H^{\infty}(D)$.

We will return to the surjectivity property towards the end of this section. We now concentrate on boundedness. Recall the Banach-Steinhaus theorem for Banach spaces: a family of linear continuous operators on a Banach space $X$, which is pointwise bounded on a set of second category, is bounded.

Let $V$ be a normed linear space. Motivated by the Banach-Steinhaus theorem, we say that $A \subset V$ has the boundedness property if every family of linear continuous operators on $V$, which is pointwise bounded on $A$, is bounded. More generally, if $U$ is a normed space and $\mathscr{A}$ is a subset of $\mathscr{L}(V, U)$, we say that $A$ has the $\mathscr{A}$-restricted boundedness property if every family of linear continuous operators in $\mathscr{A}$, which is 
pointwise bounded on $A$, is bounded. In the latter definition, if $\mathscr{A}$ is the space of adjoint operators between duals, we use the term $w^{*}$-boundedness property.

From the Banach-Steinhaus theorem, we conclude that every second category set $A$ in a Banach space has the boundedness property. However, the Nikodỳm-Grothendieck boundedness theorem (cf. [3, page 14] or [2, page 80]) says, in our terminology, that the set of characteristic functions in the unit sphere of $B(\Sigma)$ has the boundedness property. This set is certainly not of the second category, it is even nowhere dense.

We will have a look at a more recent theorem of Fernández [6] (see also [23]), which is in the same spirit as the Nikodỳm-Grothendieck theorem.

Theorem 1.3 (Fernandez' theorem). Suppose $\left(f_{n}\right)$ is a sequence in $L_{1}(T)$ such that

$$
\left|\sup _{n} \int_{T} f_{n} \phi d \theta\right|<\infty
$$

for every inner function $\phi$. Then

$$
\left|\sup _{n} \int_{T} f_{n} g d \theta\right|<\infty
$$

for every $g \in H^{\infty}(D)$ (and hence $\left(f_{n}\right)$ is bounded in the pre-dual of $H^{\infty}(D)$ ).

It is well known that the pre-dual of $H^{\infty}$ is $L_{1} / H_{0}^{1}$. Thus, by Theorem 3.5, in our language, Fernandez' theorem says that the set of inner functions has the $w^{*}$-boundedness property in $H^{\infty}(T)$. In [6, 7], the question whether the set of inner functions has the boundedness property or not was posed. In [13], it was shown that also the set of Blaschke-products has the $w^{*}$-boundedness property in $H^{\infty}(T)$, and the linear span of the Blaschke products is a first category set in $H^{\infty}(D)$.

We now give a more precise definition of the surjectivity property in normed spaces. We say that a set $A \subset X$ has the surjectivity property if, for every Banach space $Y$, every $T: Y \rightarrow X$ onto $A$ is onto $X$. If the conclusion holds for a subset $\mathscr{A} \subset \mathscr{L}(X, Y)$, we say that $A$ has the $\mathscr{A}$-restricted surjectivity property. The case where $\mathscr{A}$ is the space of adjoint operators between two duals is a special case. In this case we will say that $A$ has the $w^{*}$-surjectivity property.

We have already mentioned Seever's theorem in a particular case. We state it in full generality. Denote as usual by $B(\Sigma)$ the Banach space of bounded measurable functions on a $\sigma$-algebra $\Sigma$.

THEOREM 1.4 (Seever's theorem). Let $U$ be a Banach space and let $A$ be the subset of $B(\Sigma)$ consisting of the characteristic functions on $\Sigma$. If $T: U \rightarrow B(\Sigma)$ is such that $T U \supset A$, then $T$ is onto.

The following list is meant to sum up what we have discussed above.

(a) Second category sets have the boundedness and the surjectivity property.

(b) The set of characteristic functions in $B(\Sigma)$ has the boundedness and the surjectivity property, but is nowhere dense and its span is first category.

(c) The set of Blaschke products in $H^{\infty}(D)$ has the weak-star boundedness property and its span is first category.

(d) In Banach spaces, bounded sets have the surjectivity property if and only if they are not a countable increasing union of non-norming sets. 
(e) In this paper, it is shown that in Banach spaces, the surjectivity property and the boundedness property are equivalent. The same is true in the weak-star case, and boundedness of $A$ is not needed.

The Nikodỳm-Grothendieck theorem has interesting consequences and one might expect nice consequences of analogous theorems in other Banach spaces as well. In the following sections, we will show different techniques to obtain such theorems.

Some open questions are posed at the end of the paper. One question which is not asked, but which seems relevant, is the following: can theorems analogue to what we have found here in the normed case and in the weak-star case be proved in general situations? And how general are these situations? More specifically, what about operator spaces with pointwise topologies, or with the topology so important for the approximation property, or with the topology of uniform convergence on compact sets?

2. Some more preliminaries. One objective in this paper is to prove that in Banach spaces the $\left(w^{*}\right.$-)surjectivity property and the $\left(w^{*}\right.$-)boundedness property are equivalent to a common property, called ( $w^{*}$-)thickness. Here are some concepts we will need.

DEFINITION 2.1. Let $X$ be a normed space.

(i) A set $A \subset X$ such that

$$
\inf _{f \in S_{X^{*}}} \sup _{x \in A}|f(x)| \geq \delta
$$

for some $\delta>0$, is called norming (for $X^{*}$ ).

(ii) A set $A \subset X$ such that for all $\epsilon>0$, there exists $0<t<\infty$ such that $t A+\epsilon B_{X} \supset B_{X}$ is called almost absorbing.

(iii) A set $A \subset X$ such that there exist $0<\lambda<1$ and $0<t<\infty$ such that $t A+\lambda A \supset B_{X}$ is called $\lambda$-almost absorbing.

By the Hahn-Banach separation theorem, the following lemma is easy to prove (see Remark 2.6 for the complex case).

LEMMA 2.2. The following statements are equivalent for a set $A$ in a normed space $X$ :

(a) $A$ is norming for $X^{*}$.

(b) $\overline{\mathrm{co}}( \pm A)$ is norming for $X^{*}$.

(c) There exists a $\delta>0$ such that $\overline{\mathrm{co}}( \pm A) \supseteq \delta B_{X}$.

More specifically, we can speak about $\delta$-norming sets, where $\delta$ refers to $\delta$ in (c).

Suppose a set $B \subset X^{*}$ is such that $\inf _{x \in S_{X}} \sup _{f \in B}|f(x)| \geq \delta$ for some $\delta>0$. In this case we will call the set $B$ norming for $X$ or $w^{*}$-norming. Of course we have a similar lemma for sets which are norming for $X$.

LEMMA 2.3. The following statements are equivalent for a set $B$ in the dual $X^{*}$ of a normed space $X$ :

(a) $B$ is norming for $X$.

(b) $\overline{\mathrm{CO}}( \pm B)$ is norming for $X$.

(c) $\overline{\mathrm{CO}} w^{*}( \pm B)$ is norming for $X$.

(d) There exists a $\delta>0$ such that $\overline{\mathrm{CO}}^{w^{*}}( \pm B) \supseteq \delta B_{X^{*}}$. 
Proposition 2.4. Let $X$ be a normed space. Consider the following statements:

(a) $A$ is almost absorbing.

(b) $A$ is $\lambda$-almost absorbing.

(c) $A$ is norming.

(d) $A$ is fundamental (i.e., $\overline{\operatorname{span}} A=X$ ).

Then $(a) \Rightarrow(b) \Rightarrow(c) \Rightarrow(d)$. For convex sets $(a) \Leftrightarrow(b)$. (d) does not imply (c) even if $A$ is closed, convex, and symmetric.

Proof. The proof of $(\mathrm{a}) \Rightarrow(\mathrm{b})$ is trivial.

(b) $\Rightarrow$ (c). If $A$ is $\lambda$-absorbing, there exists $\lambda, t$ such that $B_{X} \subset t A+\lambda B_{X}$. Then, of course, $B_{X} \subset t \cdot \overline{\mathrm{co}}( \pm A)+\lambda B_{X}$. Thus,

$$
\begin{aligned}
B_{X} & \subset t \cdot \overline{\mathrm{CO}}( \pm A)+\lambda B_{X} \subset t \cdot \overline{\mathrm{CO}}( \pm A)+\lambda\left(t \cdot \overline{\mathrm{CO}}( \pm A)+\lambda B_{X}\right) \\
& \subset \cdots \subset \bar{C} \cdot \overline{\mathrm{CO}}( \pm A)+\lambda t \cdot \overline{\mathrm{CO}}( \pm A)+\cdots+\lambda^{n} t \cdot \overline{\mathrm{CO}}( \pm A)+\lambda^{n+1} B_{X} .
\end{aligned}
$$

Since $\overline{\mathrm{co}}( \pm A)$ is convex, we obtain

$$
B_{X} \subset t \cdot \overline{\mathrm{CO}}( \pm A)\left(1+\lambda+\cdots \lambda^{n}\right)+\lambda^{n+1} B_{X} .
$$

But this is true for every $n$. Hence we can take limits

$$
B_{X} \subset t \cdot \overline{\mathrm{CO}}( \pm A) \frac{1}{1-\lambda},
$$

which gives

$$
\overline{\mathrm{co}}( \pm A) \supset \frac{1-\lambda}{t} B_{X},
$$

and by Lemma 2.2, $A$ is norming.

The proof of $(c) \Rightarrow(d)$ is trivial.

We now prove that (b) implies (a) for convex sets. Let $\epsilon>0$. Since $A$ is $\lambda$-almost absorbing and convex,

$$
\begin{aligned}
B_{X} & \subset t A+\lambda B_{X} \subset t A+\lambda\left(t A+\lambda B_{X}\right) \\
& =t(1+\lambda) A+\lambda^{2} B_{X} \subset \cdots \subset\left(1+\lambda+\lambda^{2}+\cdots+\lambda^{n-1}\right) t A+\lambda^{n} B_{X},
\end{aligned}
$$

for every natural number $n$. Since $\lambda<1, \lambda^{n}$ is eventually less than $\epsilon$.

To see that (d) does not imply (c), let

$$
A=\overline{\mathrm{co}}\left( \pm \frac{e_{n}}{n}\right) \subset l_{1} .
$$

It is easy to check that $A$ has the desired properties.

Later we will need the now well-known construction of Davis, Figiel, Johnson, and Pełzcynski. See [1] or [2, pages 227-228], or [15] for some recent results. We call the space constructed by this procedure from a bounded, absolutely convex set $K$, the DFJP-space constructed on $K$.

Proposition 2.5. Let $X$ be a Banach space and let $K \subset X$ be bounded, convex, and symmetric. Let $X_{K}$ be the DFJP-space constructed on $K$. Then the natural embedding $J_{K}$ of $X_{K}$ into $X$ is an isomorphism if and only if $K$ is norming for $X^{*}$. 
Proof. If $K$ is norming for $X, \overline{J_{K}(K)} \supset \lambda \cdot B_{X}$ for some $\lambda>0$. Thus $B_{X_{K}} \overline{J_{K}\left(B_{X_{K}}\right)} \supset$ $\lambda \cdot B_{X}$ and $J_{K}$ is invertible.

If $X_{K}$ and $X$ are isomorphic by $J_{K}$ then, for some $\delta>0$,

$$
\delta B_{X} \subset C \stackrel{\text { def }}{=} J_{K}\left(B_{X_{K}}\right) .
$$

But, by the construction of $X_{K}$ (see [15]),

$$
C \subset a^{n} K+a^{-n} B_{X}
$$

for all $n$. We use that $\delta B_{X} \subset C$ inductively for a constant $n$ :

$$
\begin{aligned}
a^{n} K+a^{-n} B_{X} & \subset a^{n} K+\frac{a^{-n}}{\delta}\left(a^{n} K+a^{-n} B_{X}\right) \subset\left(a^{n}+\frac{1}{\delta}\right) K+\frac{a^{-2 n}}{\delta} B_{X} \\
& \subset\left(a^{n}+\frac{1}{\delta}\right) K+\frac{a^{-2 n}}{\delta^{2}}\left(a^{n} K+a^{-n} B_{X}\right) \subset\left(a^{n}+\frac{1}{\delta}+\frac{a^{-n}}{\delta^{2}}\right) K+\frac{a^{-3 n}}{\delta^{2}} B_{X} .
\end{aligned}
$$

Continuing this way gives after $r$ steps

$$
a^{n} K+a^{-n} B_{X} \subset a^{n} K+\left\{\frac{1}{\delta} \sum_{k=0}^{r-1} \frac{1}{\left(\delta \cdot a^{n}\right)^{k}}\right\} K+\left(\frac{1}{\delta a^{n}}\right)^{r} \cdot a^{-n} B_{X} .
$$

This is true for any $n$. Now choose $N$ so big that $\delta a^{N}>2$. Then,

$$
B_{X} \subset \frac{2\left(1-(1 / 2)^{r}\right) 2^{N}}{\delta} K+\frac{1}{2^{r}} B_{X} .
$$

Now, by letting $r \rightarrow \infty$, we obtain

$$
\frac{\delta}{2 a^{N}} B_{X} \subset \bar{K}
$$

This proves that $K$ is norming.

REMARK 2.6. When the space under consideration is complex, a norming set $A$ is a set such that for some $\delta>0, \overline{\mathrm{co}}\left(\cup_{|r|=1} r A\right) \supset \delta B_{X}$. It is easy to verify that all the results so far are true with complex scalars instead of real scalars.

REMARK 2.7. Note that a set $A$ has the surjectivity property (boundedness property) if and only if $\operatorname{co}\left(\cup_{|r|=1} r A\right)$ has the surjectivity property (boundedness property).

Fundamental sets are useful when testing for weak-star and weak convergence of nets. The following proposition is well known and classic.

Proposition 2.8. Let $X$ be a normed space.

(a) Suppose a bounded net in $X^{*}$ converges pointwise on a fundamental set $A \subset X$. Then it converges in the weak-star topology.

(b) Suppose a bounded net in $X$ converges pointwise on a fundamental set $A \subset X^{*}$. Then it converges weakly. 
REMARK 2.9. The Simons-Rainwater theorem (cf. [24]) is trivial (and works also for bounded nets) whenever the James boundary under consideration is fundamental in $X^{*}$ (e.g., when $X^{*}$ has the RNP). If we have a sequence, an interesting question is to give conditions on the fundamental set such that boundedness automatically follows from the pointwise convergence of the sequence.

EXAMPLE 2.10. A famous theorem of Marshall says: $H^{\infty}(T)$ is the closed, linear span of the Blaschke products. In other words, the Blaschke products form a fundamental subset of $H^{\infty}$. Later it has been shown that the Blaschke products form a subset of $H^{\infty}$ which is 1-norming for the dual (see [10, pages 195-197]). The most recent theorem in this direction is, as far as I know, the result from [19] saying that the interpolating Blaschke products form a set which is $10^{-7}$-norming for the dual.

We now define the terms "thick" and "thin."

DEFINITION 2.11. A set is called ( $\left.w^{*}\right)$-thin if it can be written as a countable increasing union of $\left(w^{*}\right)$-non-norming sets. A set which is not $\left(w^{*}\right.$-)thin is called ( $w^{*}$-)thick.

This classification of sets is not standard and the terms thick and thin sets are often used to describe properties of sets. Maybe it would be better to call thick sets Fonf sets, since I think he is the one who first and best demonstrated the relevance and importance of the thick sets. Fonf, however, never uses the word thick, but in his works he always operates with "thin" and "not thin" sets. See, for example, [8, 9, 14] for examples of earlier use and applications of these concepts.

EXAMPLE 2.12. To get an idea of these properties, one can think of the extreme points $A$ of the unit ball in $l_{1}$. This is a countable, hence thin (and thus $w^{*}$-thin) set. The set $B_{l_{1}}$ is the norm-closure of the convex hull of its extreme points $A=\operatorname{ext} B_{l_{1}}=$ $\left\{ \pm e_{i}\right\}_{i=1}^{\infty}$ so it is 1-norming. Let $f_{n}=n e_{n}$. Then $\left\{f_{n}\right\} \subset c_{0}$ is pointwise bounded on $A$, but obviously not bounded as a subset of $c_{0}$. So this $A$ does not have the $w^{*}$-boundedness property. Now define an operator $T \in L\left(l_{\infty}, l_{1}\right)$ by $T\left(x_{1}, x_{2}, \ldots\right)=$ $\left(2^{-n} x_{n}\right)_{n=1}^{\infty}$. Then $T$ is onto $A$, but since it is injective, it cannot be onto $l_{1}$. Thus $A$ does not have the surjectivity property. Since $T$ is the adjoint of $S: c_{0} \rightarrow l_{1}$ defined by $S\left(x_{1}, x_{2}, \ldots\right)=\left(2^{-n} x_{n}\right)_{n=1}^{\infty}$, $A$ does not have the $w^{*}$-surjectivity property either.

We want to show that the simple Example 2.12 is just a special case of a very general principle in Banach spaces.

3. The boundedness property in normed spaces. We recall the definition of the boundedness property.

Definition 3.1. A subset $A$ of a normed linear space $X$ is said to have the boundedness property if for every normed space $Y$, every family $\left(T_{\alpha}\right) \subset \mathscr{L}(X, Y)$, which is pointwise bounded on $A$, is bounded.

A special variant of the following theorem was first published in [18]. Also parts of it are implicit in [9].

THEOREM 3.2. Suppose A is a subset of a normed space $X$. The following statements are equivalent: 
(a) A has the boundedness property.

(b) Every sequence $\left(f_{n}\right) \subset X^{*}$ which is pointwise bounded on $A$ is a bounded sequence in $X^{*}$.

(c) $A$ is thick.

Proof. (a) clearly implies (b). To prove that (b) implies (c) suppose $A$ is thin. Then we can pick a countable, increasing covering, $\cup A_{n}$, of $A$, consisting of sets which are non-norming for $X^{*}$. Thus, we can find a sequence $\left(f_{n}\right) \subset X^{*}$ such that $f_{n} \in n S_{X^{*}}$ but $\sup _{A_{n}}\left|f_{n}(x)\right|<1$. Let $x$ be an arbitrary element of $A$. Then there is a natural number $m$ such that $x \in A_{m}$. Thus, since $\left(A_{n}\right)$ is increasing, then

$$
\left|f_{k}(x)\right| \leq\left\|f_{k}\right\|\|x\|<m\|x\| \quad \text { if } k<m,
$$

while

$$
\left|f_{k}(x)\right|<1 \text { if } k \geq m .
$$

This proves that (b) implies (c).

To show that (c) implies (a), suppose $A$ is thick and ( $\left.T_{\alpha}\right)$ is pointwise bounded on $A$, that is,

$$
\sup _{\alpha}\left\|T_{\alpha} x\right\|<\infty \quad \forall x \in A .
$$

Put $A_{m}=\left\{x \in A: \sup _{\alpha}\left\|T_{\alpha} x\right\| \leq m\right\}$. Then $\left(A_{m}\right)$ is an increasing family of sets which covers $A$. Since $A$ is thick, some $A_{q}$ is norming. Then, using Lemma 2.2, there exists a $\delta>0$ such that

$$
\overline{\mathrm{CO}}\left( \pm A_{q}\right) \supseteq \delta B_{X}
$$

But then, for arbitrary $\alpha$,

$$
\delta\left\|T_{\alpha}\right\|=\sup _{x \in \delta S_{X}}\left\|T_{\alpha} x\right\| \leq \sup _{x \in \overline{\mathrm{co}}\left( \pm A_{q}\right)}\left\|T_{\alpha} x\right\| \leq q .
$$

Thus $\sup _{\alpha}\left\|T_{\alpha}\right\| \leq q / \delta<\infty$, and the theorem is proved.

REMARK 3.3. Note how simple and general Theorem 3.2 is. It gives an interesting perspective on the classical Banach-Steinhaus theorem for Banach spaces, as soon as we have shown that sets of second category are thick. This is done in the following easy lemma.

LEMMA 3.4. Let $X$ be a normed space and suppose $A$ is a second category set in $X$. Then $A$ is thick.

Proof. Suppose $A$ is covered by an increasing family $\left(A_{i}\right)$. Since $A$ is of second category, some $\overline{A_{m}}$ contains a ball. Then $\overline{\mathrm{co}}\left( \pm A_{m}\right)$ contains a ball centered at the origin, and hence $A_{m}$ is norming. Since $\left(A_{i}\right)$ is arbitrary, $A$ must be thick.

We also have a "uniform boundedness theorem" characterizing $w^{*}$-thickness.

THEOREM 3.5. Let $X$ be a normed space and suppose $B$ is a subset of $X^{*}$. Then the following statements are equivalent:

(a) B has the $w^{*}$-boundedness property. 
(b) Every sequence $\left(x_{n}\right) \subset X$ which is pointwise bounded on $A$ is a bounded sequence in $X$.

(c) $B$ is $w^{*}$-thick.

Proof. (a) of course implies (b). The proof that (b) implies (c) is completely analogous to the corresponding part of the proof of Theorem 3.2. The proof that (c) implies (a) is also similar to the corresponding part of the proof of Theorem 3.2. Just put

$$
B_{m}=\left\{x^{*} \in B: \sup _{\alpha}\left\|T_{\alpha}^{*} x^{*}\right\| \leq m\right\},
$$

and use the $w^{*}$-continuity of $T_{\alpha}^{*}$.

REMARK 3.6. The set of extreme points of the unit ball of $l_{1}$ shows that a set can be norming for the dual without being $w^{*}$-thick. It is also possible for a set to be $w^{*}$-thick without being norming for the dual (although it is of course norming for the pre-dual). In fact, the unit ball of any non-reflexive space, considered as a subset of the bidual, give examples of such situations (they are not even fundamental).

COROLlaRY 3.7. The Blaschke products in $H^{\infty}(D)$ is $w^{*}$-thick and 1-norming for the dual.

Proof. By the main result in [13], the set of Blaschke products satisfies Theorem 3.5(b). The set is 1-norming by [10, Corollary 2.6, page 196].

4. The surjectivity property in Banach spaces. In this section, we study the surjectivity property in Banach spaces. We start with the formal definition.

DEFINITION 4.1. In a normed linear space $X$ a set $A$ is said to have the surjectivity property if for every normed linear space $Y$, every $T \in \mathscr{L}(Y, X)$, such that $T Y \supset A$, is onto $X$. If $A$ in a normed space has the surjectivity property for all $T$ 's coming from Banach spaces, we say that $A$ has the surjectivity property for Banach spaces.

Recall that an operator $T: Y \rightarrow X$ is called Tauberian if $\left(T^{* *}\right)^{-1}(X) \subset Y$. As an intuition, it is often helpful to think of these operators as opposite to weakly compact operators. A nice reference for the theory of Tauberian operators is [11].

The next theorem shows the connection between thickness and the surjectivity property. The theorem is a generalization of Theorem 1.1, discovered by Kadets and Fonf [14].

THEOREM 4.2. Suppose $A$ is a subset of a Banach space $X$. The following statements are equivalent:

(a) A has the surjectivity property for Banach spaces.

(b) For every Banach space $Y$, every injection $T: Y \rightarrow X$, which is onto $A$, is an isomorphism.

(c) For every Banach space $Y$, every Tauberian injection $T: Y \rightarrow X$, which is onto A, is an isomorphism.

(d) $A$ is thick.

Proof. Of course (a) implies (b) and (b) implies (c). 
To show that (c) implies (d), suppose (d) is not true, that is, $A$ is thin. We will construct a Tauberian injection which is onto $A$ but not onto all of $X$. Let $\left(A_{i}\right)$ be an increasing family of non-norming subsets of $A$ such that $A=\cup_{i=1}^{\infty} A_{i}$. Since $\cup_{i=1}^{\infty} A_{i}=$ $\cup_{i=1}^{\infty} A_{i} \cap i \cdot B_{X}$ and $A_{i} \cap i \cdot B_{X}$ is non-norming, we may assume each $A_{i}$ to be contained in $i \cdot B_{X}$. Put $C_{1}=A_{1}$ and $C_{i}=A_{i} \backslash A_{i-1}$. Define

$$
C=\overline{\mathrm{CO}}\left( \pm \bigcup_{i=1}^{\infty} \frac{C_{i}}{i^{2}}\right) .
$$

Then $C$ is closed, bounded, convex, and symmetric. We now show that $C$ is nonnorming for $X^{*}$. To do this, let $\epsilon>0$ and take $j$ such that $1 / j<\epsilon$. Since $A_{j}$ is not a norming set, there is a functional $f \in S_{X^{*}}$ such that $\sup _{x \in A_{j}}|f(x)|<\epsilon$. By the definition of $C$,

$$
\sup _{x \in C}|f(x)|=\sup _{i}\left\{\frac{1}{i^{2}} \sup _{x \in C_{i}}|f(x)|\right\} .
$$

Take an arbitrary $y \in C$. Then either $y \in A_{i}, i \leq j$ or $y \in A_{i}, i>j$. In the first case

$$
|f(y)| \leq \sup _{x \in A_{j}}|f(x)|<\epsilon .
$$

In the second case, since $C_{i} \subset i \cdot B_{X}$,

$$
|f(y)| \leq \sup _{i>j}\left\{\frac{1}{i^{2}} \sup _{x \in C_{i}}|f(x)|\right\} \leq \sup _{i>j}\left\{\frac{1}{i^{2}} \cdot i\right\} \leq \frac{1}{j}<\epsilon .
$$

Thus,

$$
\sup _{x \in C}|f(x)|<\epsilon
$$

and $C$ is not norming for $X^{*}$.

Hence, by Proposition 2.5, the Davis-Figiel-Johnson-Pelzcynski construction on $C$ will produce a Banach space $Y$ and an operator $J: Y \rightarrow X$ with the desired properties, that is, it is injective, Tauberian, onto $A$ but not onto all of $X$.

It remains to show that (d) implies (a). To do this, let $T$ be any bounded, linear operator, from a Banach space $Y$, into $X$ and onto $A$. Put $A_{i}=T\left(i \cdot B_{Y}\right) \cap A$, where $Y$ is the domain space of $T$. Since $T$ is onto $A,\left(A_{i}\right)_{i=1}^{\infty}$ is an increasing covering of $A$. Since $A$ is thick, some $A_{j}$ is norming for $X^{*}$. By Lemma 2.2, there exists a $\delta>0$ such that

$$
j \cdot \overline{\mathrm{CO}}\left( \pm T B_{Y}\right)=j \cdot \overline{T B_{Y}} \supset \delta B_{X} .
$$

Hence $\overline{T B_{Y}} \supset(\delta / j) \cdot B_{X}$ and by, for example, [20, Theorem 4.13], $T$ is onto.

We now combine Theorems 3.2 and 4.2 to obtain our main result.

COROLLARY 4.3. In Banach spaces, the surjectivity property for Banach spaces and the boundedness property are both equivalent to thickness.

REMARK 4.4. By the Nikodỳm-Grothendieck boundedness theorem the characteristic functions is a thick set in $B(\Sigma)$. Thus Seever's theorem (see Theorem 1.4) follows as a special variant of the very general Theorem 4.2 . 
REMARK 4.5. The DFJP-embedding $J$ is "a little more" than Tauberian because $J^{* *}$ is Tauberian. In [15] an easy argument is given to show that, in fact, $J$ is a norm-norm homeomorphism when restricted to the set on which the DFJP-space is constructed.

It is interesting to compare Theorem 4.2 with the following observation of Neidinger [16, page 119]. Its proof is given by a close inspection of a standard proof of the open mapping theorem, see for example [20, page 48]. An interesting application can be found in [17].

LEMmA 4.6 (R. Neidinger). Let $X$ and $Y$ be Banach spaces and let $T \in \mathscr{L}(X, Y)$. Then the following statements are equivalent:

(a) $T B_{X}$ almost $\lambda$-absorbs $B_{Y}$.

(b) $T B_{X}$ almost absorbs $B_{Y}$.

(c) $T B_{X}$ absorbs $B_{Y}$.

(d) $T$ is onto.

An analogue to Lemma 4.6, using the terms thick set and norming set, is the following.

LEMmA 4.7. Let $X$ and $Y$ be Banach spaces and let $T \in \mathscr{L}(X, Y)$. Then the following statements are equivalent:

(a) $T B_{X}$ is norming for $Y^{*}$.

(b) $T B_{X}$ is thick in $Y$.

(c) $T$ is onto.

Note that, by Proposition 2.4, (a) as well as (b) of Lemma 4.6 is equivalent to (a) of Lemma 4.7.

Proof. Only the implication $(\mathrm{a}) \Rightarrow(\mathrm{b})$ needs proof. $T B_{X}$ is norming, so there is a $\delta_{1}>0$ such that $\overline{T B_{X}} \supset \delta_{1} B_{Y}$. Suppose $T B_{X}$ has been written as an increasing countable union, $T B_{X}=\cup_{i=1}^{\infty} A_{i}$. Then $B_{X}=\cup_{i=1}^{\infty}\left(T^{-1}\left(A_{i}\right) \cap B_{X}\right)=\cup_{i=1}^{\infty} B_{i}$, an increasing union. Since $B_{X}$ is thick, there exists a number $m$ and a $\delta_{2}>0$ such that $\overline{\mathrm{co}}\left( \pm B_{m}\right) \supset \delta_{2} B_{X}$. Thus

$$
\overline{\mathrm{CO}}\left( \pm A_{m}\right)=\overline{\mathrm{CO}}\left( \pm T B_{m}\right) \supset T \overline{\mathrm{CO}}\left( \pm B_{m}\right) \supset \delta_{2} \overline{T B_{X}} \supset \delta_{1} \delta_{2} B_{Y}
$$

We now consider $w^{*}$-thick sets. Here is a characterization of such sets in terms of surjectivity properties.

TheOrem 4.8. Suppose $B$ is a subset of a dual space $X^{*}$. Then the following statements are equivalent:

(a) $B$ has the surjectivity property for all dual operators into $X^{*}$, that is, $B$ has the $w^{*}$-surjectivity property.

(b) $B$ has the surjectivity property for all dual injections into $X^{*}$.

(c) $B$ is $w^{*}$-thick.

Proof. The proof that (a) implies (b) is trivial. To show that (b) implies (c), we make necessary adjustments in the corresponding proof of Theorem 4.2. First substitute $A$ 's with $B$ 's. Then define $C$ by $w^{*}$-closure. Note that $C$ is now a non- $w^{*}$-norming set. Define $Y$ to be the $w^{*}$-closure of $\operatorname{span} C$. 
If $Y \neq X^{*}$ let $T$ be the embedding of $Y$ into $X^{*}$. Then, since $Y$ is $w^{*}$-closed, $Y=$ $(X / M)^{*}$, where $M$ is the annihilator of $Y$ in $X$. Moreover, $T$ is the adjoint of the quotient map $q: X \rightarrow X / M$.

If $Y=X^{*}$ the set $C$ can be used to define a new norm $\|\cdot\|_{C}$ on $X$ by the formula

$$
\|x\|_{C}=\sup _{c \in C}|c(x)| .
$$

Then, by the definition of $C,\|\cdot\|_{C}$ is strictly weaker than the original norm. Let $E$ be the completion of $X$ in this weaker norm, let $j$ be the embedding of $X$ into $E$. The adjoint $T$ of $j$ is then continuous and injective (since $j$ is continuous and dense). Moreover, $T$ is by definition onto $C$ and hence onto $B$. Thus (b) implies (c).

To show that (c) implies (a), mimicking the corresponding proof of Theorem 4.2 gives the existence of a natural number $j$ such that

$$
{\overline{\left(T B_{Y}\right)}}^{w^{*}} \supset \frac{\delta}{j} B_{X^{*}}
$$

Now we use that $T$ is an adjoint operator. This gives us that the set $T B_{Y}$ is $w^{*}$-compact, and hence

$$
T B_{Y} \supset \frac{\delta}{j} B_{X^{*}}
$$

which concludes the proof.

The following corollary is known from [9].

Corollary 4.9. Suppose $B$ is a bounded subset of a dual space $X^{*}$. Then the following statements are equivalent:

(a) There exists a Banach space $Y$ and an injection $T: X \rightarrow Y$ such that $T^{*}$ is injective and $T^{*} Y^{*} \supset B$, but $T$ is not invertible.

(b) $B$ is $w^{*}$-thin.

COROLLARY 4.10. In the Banach space setting, the $w^{*}$-surjectivity property and the $w^{*}$-boundedness property are both equivalent to $w^{*}$-thickness.

By the Fernandez-Hui-Shapiro theorems (see Theorem 1.3 and the comments after it), the set of Blaschke-products is a $w^{*}$-thick, 1-norming subset of $H^{\infty}$. Thus, if $T$ is an adjoint operator from a dual Banach space into $H^{\infty}$ which is onto the set of Blaschke products, then $T$ is onto $X$. We state this in an alternative form.

THEOREM 4.11. Let $X$ be an arbitrary Banach space. Let $B$ denote the set of Blaschkeproducts in $H^{\infty}$. Suppose $S \in \mathscr{L}\left(L_{1} / H_{0}^{1}, X\right)$ is such that $S^{*}\left(X^{*}\right) \supset B$. Then $X$ contains $L_{1} / H_{0}^{1}$ as a closed subspace.

Proof. Since $S^{*}$ is dense, $S$ is $1-1$. Since $B$ is weak-star thick $S^{*}$ is onto. Thus $S$ has closed range.

5. The Seever property and the Nikodỳm property. In [3, Example 5, page 18] an example is given to show that the Nikodym-Grothendieck boundedness theorem may fail when the measures are not defined on a $\sigma$-algebra, but just on an algebra. 
In [22] five properties for algebras $\mathscr{A}$ of sets are discussed. They are as follows:

(i) $\mathscr{A}$ has the Vitali-Hahn-Saks property (VHS) if the Vitali-Hahn-Saks theorem holds on $\mathscr{A}$.

(ii) $\mathscr{A}$ has the Nikodỳm property $(\mathrm{N})$ if the Nikodỳm-Grothendieck boundedness theorem holds on $\mathscr{A}$.

(iii) $\mathscr{A}$ has the Orlicz-Pettis property (OP) if, for every Banach space $X$, weak countably additive $X$-valued measures are countably additive.

(iv) $\mathscr{A}$ has the Grothendieck property (G) if $B(\mathscr{A})$ is a Grothendieck space, that is, if every weak-star convergent sequence in the dual is weakly convergent.

(v) $\mathscr{A}$ has the Rosenthal property (R) if $B(\mathscr{A})$ is a Rosenthal space, that is, if every continuous, non-weakly compact, linear operator into a Banach space $X$ fixes a copy of $\ell_{\infty}$.

A $\sigma$-algebra has all these properties. It is shown in different papers (see $[4,22]$ for references) that (VHS) $\Leftrightarrow(\mathrm{N})$ and $(\mathrm{G})$, that $(\mathrm{G}) \Rightarrow(\mathrm{OP})$, that $(\mathrm{R}) \Rightarrow(\mathrm{G})$, and that no other implications hold. For sometimes it was open whether $(\mathrm{G})$ alone might imply $(\mathrm{N})$ or not. A counterexample was given by Talagrand in [25].

We say that an algebra has the Seever property (S) if Seever's theorem works on $\mathscr{A}$.

THEOREM 5.1. The following statements about an algebra Ad are equivalent:
(a) A has the Nikodym property (N).
(b) $A$ has the Seever property (S).
(c) The set $\left\{\chi_{A}: A \in \mathscr{A}\right\}$ is thick.

6. Some results on thickness in $\mathscr{L}(X, Y)^{*}$. Let $X$ and $Y$ be Banach spaces. A very useful set in $\mathscr{L}(X, Y)^{*}$ is the tensor product $X^{* *} \otimes Y^{*}$. Recall that the action of a functional $x^{* *} \otimes y^{*}$ on an operator $T \in \mathscr{L}(X, Y)$ is defined by $\left(x^{* *} \otimes y^{*}\right)(T)=x^{* *}\left(T^{*} y^{*}\right)$. In [12, Lemma 1.7b, page 268] it is shown that ext $B_{X^{* *}} \otimes \operatorname{ext} B_{Y^{*}}$ is 1-norming for $L(X, Y)$. It needs not to be $w^{*}$-thick. But, often, it is.

LEMMA 6.1. Suppose $A$ and $B$ are $w^{*}$-thick subsets of $X^{* *}$ and $Y^{*}$, respectively. Then $A \otimes B$ is a $w^{*}$-thick subset of $\mathscr{L}(X, Y)^{*}$.

Proof. We will use Theorem 3.5. Let $\left(T_{n}\right)$ be a sequence in $L(X, Y)$ such that

$$
\sup _{n}\left|x^{* *} \otimes y^{*}\left(T_{n}\right)\right|=\sup _{n}\left|x^{* *}\left(T_{n}^{*} y^{*}\right)\right|<\infty
$$

for all $x^{* *} \in A$ and all $y^{*} \in B$. Since $A$ is $w^{*}$-thick we conclude that

$$
\sup _{n}\left\|\left(T_{n}^{*} y^{*}\right)\right\|<\infty
$$

for all $y^{*} \in B$. Since $B$ is $w^{*}$ - thick,

$$
\sup _{n}\left\|T_{n}^{*}\right\|<\infty
$$

and the result follows. 
Since, by definition, no countable set can be $w^{*}$-thick, the extreme points of $B_{l_{1}}$ is a $w^{*}$-thin set. This is in fact a special case of a rather difficult theorem discovered by V. P. Fonf. Recall that a James boundary $J$ for $X$ is a subset of $X^{*}$ such that every $x \in X$ attains its norm on $J$. As an example, the set of extreme points of the dual unit ball is always a James boundary for $X$.

THEOREM 6.2. If a Banach space $X$ admits a $w^{*}$-thin James boundary $J$, then $X$ contains a copy of $c_{0}$.

We only present a list to show how the theorem can be proved with the help of different papers. The list points to the simplest proof known to the author.

PRoof. (a) Note that the restriction of a James boundary to a subspace $Y$ is a James boundary for $Y$.

(b) Put $J=\cup_{n} A_{n}$. By Simons' generalization of the Rainwater lemma [24], there is a sequence $\left(x_{n}\right)$ on $S_{X}$ which converges weakly to 0 . By the Bessaga-Pełzcynski selection principle (cf. [2, page 42]) $\left(x_{n}\right)$ can be assumed to be a basic sequence. Let $Y=\left[x_{n}\right]$. We look for $\mathcal{c}_{0}$ inside $Y$.

(c) Let $T$ be the natural embedding of $Y$ into $X$. Put $B_{n}=T^{*}\left(A_{n}\right)$. Then show that $J^{\prime}=\cup B_{n}$ is a James boundary for $Y$.

(d) Show that each $B_{n}$ is relatively norm-compact as done in [8, page 489]. Thus $Y$ has a $\sigma$-compact James boundary $J^{\prime}$.

(e) Use [5, Lemma 27] to renorm $Y$ equivalently to have a countable James boundary $J^{\prime \prime}$.

(f) Follow the proof of [5, Theorem 23] to construct a copy of $c_{0}$ inside a once more equivalently renormed version of $Y$. This copy is also a copy in $X$.

An interesting result follows from Lemma 6.1 and Theorem 6.2.

Corollary 6.3. Suppose $X^{*}$ and $Y$ does not contain a copy of $c_{0}$. Then the set $E=\operatorname{ext} B_{X^{* *}} \otimes \operatorname{ext} B_{Y^{*}}$ is $w^{*}$-thick in $L(X, Y)^{*}$.

Proof. Since neither $X^{*}$ nor $Y$ contains a copy of $c_{0}$, the sets ext $B_{X^{* *}}$ and ext $B_{Y^{*}}$ are both $w^{*}$-thick. Hence, by Lemma 6.1, $E$ is $w^{*}$-thick.

REMARK 6.4. Note that the set $E$ is not necessarily a James boundary for $L(X, Y)$. But being "identical" to the set ext $B_{K(X, Y) *}$ it is a James boundary for $K(X, Y)$.

By combining the main result from [9] with the knowledge of the exposed points of the dual unit ball of $K(X, Y)$ (cf. [21, Theorem 5.1]), we obtain the following theorem on $w^{*}$-thickness of $\exp B_{K(X, Y)^{*}}$.

THEOREM 6.5. Suppose $X^{*}$ and $Y$ are separable and $Y$ does not contain a copy of $c_{0}$. Then $\exp B_{K(X, Y) *}$ is $w^{*}$-thick.

Proof. Since $X^{*}$ is a separable dual, it has the RNP and thus does not contain a copy of $c_{0}$. By the main result from [9], the sets $A=\exp B_{X^{* *}}$ and $B=\exp B_{Y^{*}}$ are both $w^{*}$-thick. Hence, by Lemma $6.1 A \otimes B$ is $w^{*}$-thick. But, by [21], $A \otimes B$ is exactly the set of exposed points of $B_{K(X, Y) *}$. 
REMARK 6.6. When $X^{*}$ and $Y$ both are separable we obtain that $K(X, Y)$ is separable. However, $K(X, Y)$ may very well contain $c_{0}$ even though $X^{*}$ and $Y$ does not. For example, the space $K\left(l_{2}\right)$ contains a copy of $c_{0}$.

COROLlary 6.7. Suppose $X$ and $Y$ are separable, reflexive spaces. Then $w^{*}$ $\exp B_{K(X, Y) *}$ is $w^{*}$-thick.

Proof. The result follows since $w^{*}$-exp $B_{K(X, Y)^{*}}=w^{*}$-exp $B_{X} \otimes w^{*}$-exp $B_{Y^{*}}$.

COROLLARY 6.8. Suppose $X$ and $Y$ are separable, reflexive spaces. Then every James boundary of $K(X, Y)$ is $w^{*}$-thick.

Proof. Every James boundary must contain the $w^{*}$-exposed points of $B_{X^{*}}$.

7. Some questions and remarks. Suppose a Banach space contains a thin, fundamental set. Then, by the definition of such a set, there exists a $w^{*}$-null sequence $\left(x_{n}^{*}\right)$ on $S_{X^{*}}$. Thus, the Josefsson-Nissenzweig theorem is just a triviality in such spaces. Of course, every separable Banach space contains a thin, even norming, set (take any dense countable subset of $B_{X}$ ). Also, it is immediate that any Banach space containing a complemented separable subspace contains a thin, norming set. Thus, WCG spaces contain thin, norming sets.

QUESTION 7.1. Does every Banach space contain a fundamental, thin set? Does every Banach space contain a norming, thin set?

Fernandez, Hui, and Shapiro have asked (in our notation) whether the Blaschkeproducts is a thick subset in $H^{\infty}$ (not only $w^{*}$-thick). We formulate an extended question:

QUESTION 7.2. Is the set of inner functions (Blaschke-products) a thick subset in $H^{\infty}$ ? Is the set of interpolating Blaschke-products thick or $w^{*}$-thick?

By a theorem of Mooney (see [10, pages 206-207]), the pre-dual of $H^{\infty}$ is weak sequentially complete. Thus, it does not contain a copy of $c_{0}$. Hence, any James boundary in $H^{\infty}$ is $w^{*}$-thick. In light of V. P. Fonf's theorem (Theorem 6.2) it is natural to ask:

QUESTION 7.3. Is the set of inner functions (Blaschke-products, interpolating Blaschke-products) a James boundary in $H^{\infty}$ ?

If so, Fernandez' and Shapiro's results would follow as special cases of Theorem 6.2. We end this paper by giving a list of sets for which results on thickness are known.

THEOREM 7.4. The following results on thickness are valid:

(a) Any James-boundary of a Banach space not containing $c_{0}$ is $w^{*}$-thick (see [8]).

(b) If $X$ is a separable Banach space not containing $c_{0}$, then $\exp B_{X^{*}}$ is $w^{*}$-thick (see [9]).

(c) The set of characteristic functions in $B(\mathscr{A})$ when $\mathscr{A}$ has the Nikodỳm (Seever) property, is thick (Nikodym-Grothendieck [3]). Thus, ext $B_{l_{\infty}}$ is thick. 
(d) The set of inner functions and Blaschke products in $H^{\infty}$ are $w^{*}$-thick and norming for the dual (see $[6,13])$.

(e) The tensor product of two $w^{*}$-thick sets in $X^{* *}$ and $Y^{*}$ is a $w^{*}$-thick subset in $L(X, Y)^{*}$.

(f) Suppose $X$ and $Y$ are separable, reflexive spaces. Then every James boundary of $K(X, Y)$ is $w^{*}$-thick.

\section{REFERENCES}

[1] W. J. Davis, T. Figiel, W. B. Johnson, and A. Pełczyński, Factoring weakly compact operators, J. Funct. Anal. 17 (1974), 311-327.

[2] J. Diestel, Sequences and Series in Banach Spaces, Graduate Texts in Mathematics, vol. 92, Springer-Verlag, New York, 1984.

[3] J. Diestel and J. J. Uhl Jr., Vector Measures, Mathematical Surveys, vol. 15, American Mathematical Society, Rhode Island, 1977, with a foreword by B. J. Pettis.

[4] _ Progress in vector measures-1977-83, Measure Theory and Its Applications (Sherbrooke, Que., 1982), Springer, Berlin, 1983, pp. 144-192.

[5] M. Fabian and V. Zizler, Introduction to Banach Spaces III, Matfyzpress, Prague, 1997.

[6] J. Fernández, A boundedness theorem for $L^{1} / H_{0}^{1}$, Michigan Math. J. 35 (1988), no. 2, 227 231.

[7] J. Fernández, S. Hui, and H. Shapiro, Unimodular functions and uniform boundedness, Publ. Mat. 33 (1989), no. 1, 139-146.

[8] V. P. Fonf, Weakly extremal properties of Banach spaces, Math. Notes 45 (1989), no. 6, 488-494, translated from Mat. Zametki 45 (1989), no. 6, 83-92. .

[9] _ On exposed and smooth points of convex bodies in Banach spaces, Bull. London Math. Soc. 28 (1996), no. 1, 51-58.

[10] J. B. Garnett, Bounded Analytic Functions, Academic Press, New York, 1981.

[11] M. Gonzales, Properties and applications of Tauberian operators, Extracta Math. 3 (1990), 91-107.

[12] P. Harmand, D. Werner, and W. Werner, M-Ideals in Banach Spaces and Banach Algebras, Lecture Notes in Mathematics, vol. 1547, Springer-Verlag, Berlin, 1993.

[13] S. Hui, An extension of a theorem of J. Fernández, Bull. London Math. Soc. 20 (1988), no. 1, 34-36.

[14] M. I. Kadets and V. P. Fonf, Two theorems on the massiveness of the boundary in a reflexive Banach space, Funktsional. Anal. i Prilozhen. 17 (1983), no. 3, 77-78.

[15] A. Lima and O. Nygaard, Isometric factorization of weakly compact operators and the approximation property, Israel J. Math. 119 (2000), 325-348.

[16] R. D. Neidinger, Properties of Tauberian operators on Banach spaces, Ph.D. thesis, dissertation, University of Texas at Austin, 1984.

[17] _ Factoring operators through hereditarily-l ${ }^{p}$ spaces, Banach Spaces (Columbia, Mo., 1984), Lecture Notes in Math., vol. 1166, Springer, Berlin, 1985, pp. 116-128.

[18] O. Nygaard, A strong uniform boundedness principle in Banach spaces, Proc. Amer. Math. Soc. 129 (2001), no. 3, 861-863.

[19] K. Øyma, The closed convex hull of the interpolating Blaschke products, Publ. Mat. 41 (1997), no. 2, 659-669.

[20] W. Rudin, Functional Analysis, 2nd ed., International Series in Pure and Applied Mathematics, McGraw-Hill, New York, 1991.

[21] W. Ruess, Duality and geometry of spaces of compact operators, Functional Analysis: Surveys and Recent Results, III (Paderborn, 1983), North-Holland Math. Stud., vol. 90, North-Holland, Amsterdam, 1984, pp. 59-78.

[22] W. Schachermayer, On some classical measure-theoretic theorems for non-sigma-complete Boolean algebras, Dissertationes Math. (Rozprawy Mat.) 214 (1982), 33. 
[23] H. S. Shapiro, A uniform boundedness principle concerning inner functions, J. Analyse Math. 50 (1988), 183-188.

[24] S. Simons, An eigenvector proof of Fatou's lemma for continuous functions, Math. Intelligencer 17 (1995), no. 3, 67-70.

[25] M. Talagrand, Propriété de Nikodým et propriété de Grothendieck, Studia Math. 78 (1984), no. 2, 165-171 (French).

Olav NygAard: Department of MAthematics, AgDer University College, Servicebox 422, 4604 KRISTIANSAND, NORWAY

E-mail address: olav. nygaard@ia.no 


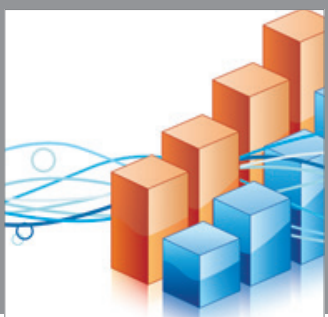

Advances in

Operations Research

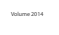

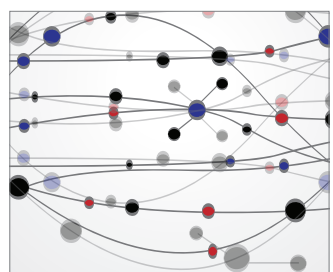

\section{The Scientific} World Journal
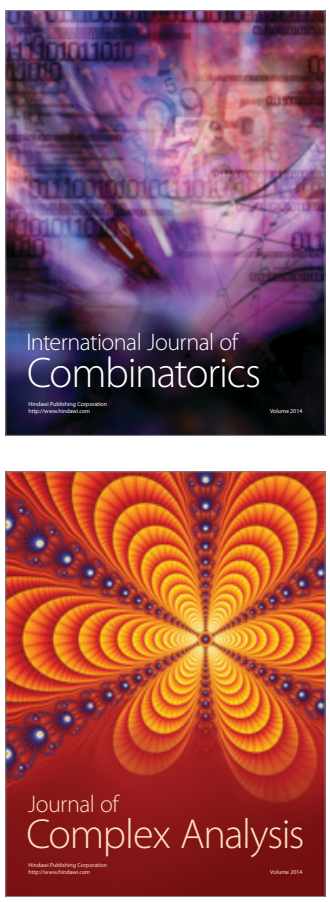

International Journal of

Mathematics and

Mathematical

Sciences
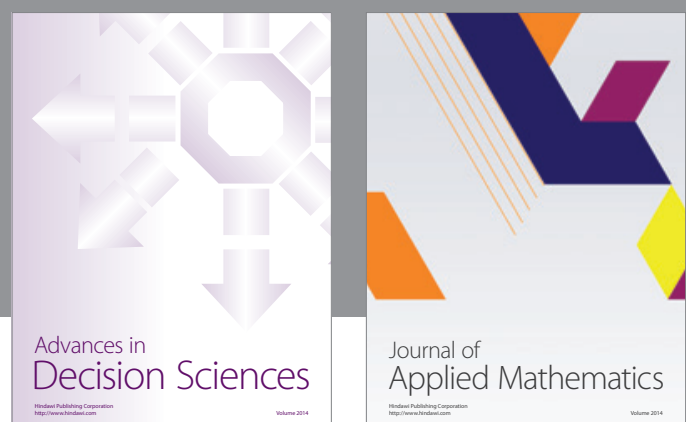

Journal of

Applied Mathematics
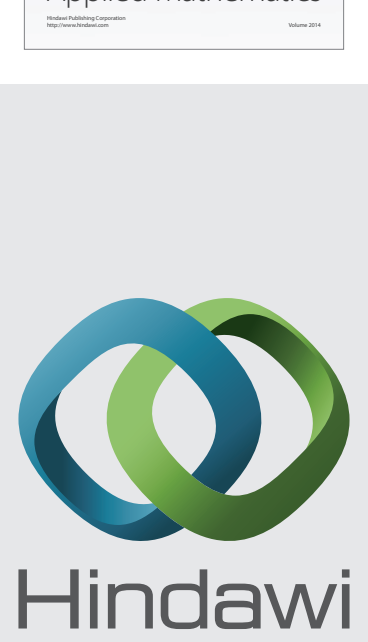

Submit your manuscripts at http://www.hindawi.com
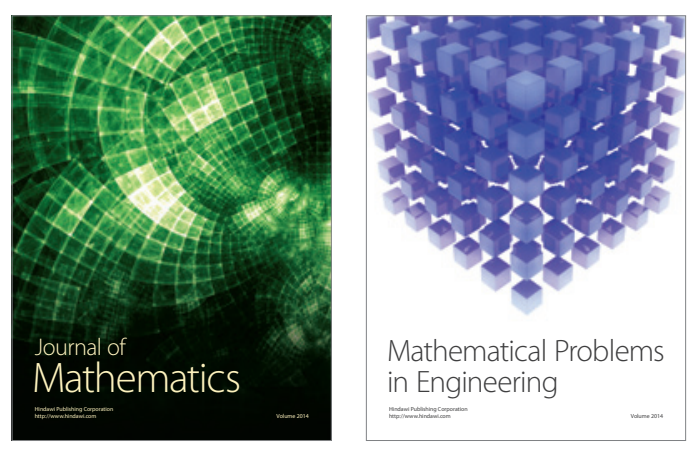

Mathematical Problems in Engineering
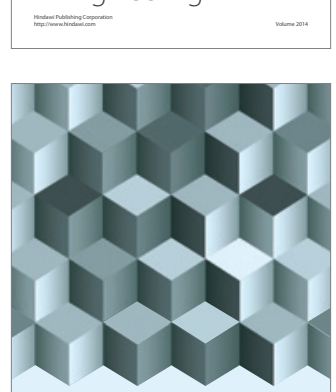

Journal of

Function Spaces
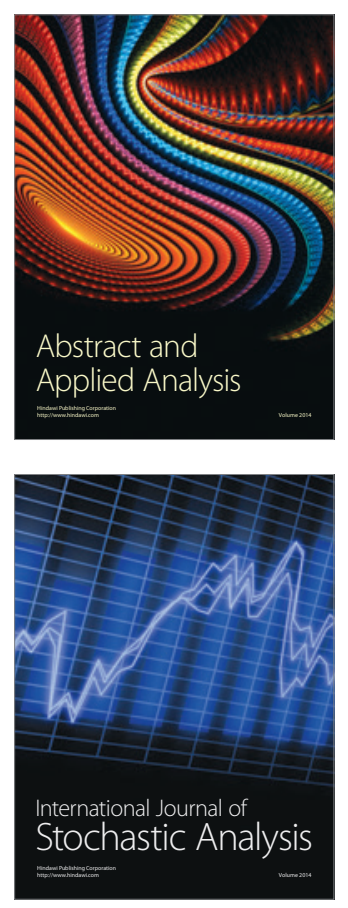

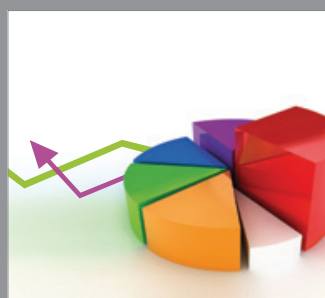

ournal of

Probability and Statistics

Promensencen
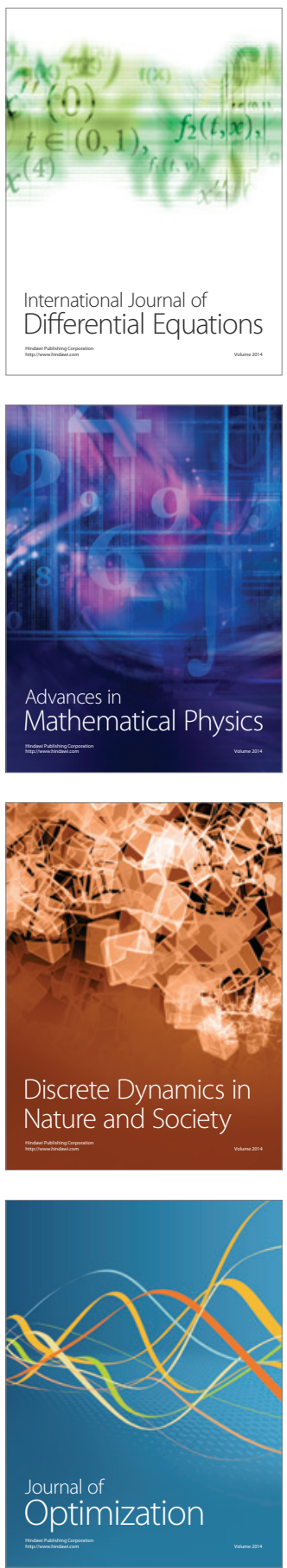